\title{
Early Lupus Project - One-year Follow-up of an Italian Cohort of Patients with Systemic Lupus Erythematosus of Recent Onset.
}

\begin{abstract}
Background: Systemic Lupus Erythematosus (SLE) is an autoimmune disease with a high degree of variability at onset, with problems for a correct and prompt diagnosis. We undertook this project with the purpose of collecting an inception cohort of Italian patients with recent (less than 12 months) onset SLE, in order to obtain information on the main clinical and serological characteristics at the beginning of the disease.

The objective of the present paper is to describe the clinical and serological features obtained after a one-year follow-up period of this inception cohort, making comparison with the information obtained at the enrollment.
\end{abstract}

Methods: All patients with a diagnosis of SLE (1997 ACR criteria) and a disease duration lower than 12 months were consecutively enrolled in a multicentre prospective study. Information on clinical and serological characteristics at study entry and then every 6 months were collected into a specific electronic database. Statistical analysis was performed using $\mathrm{R}$ statistical environment (The R project for statistical computing, https://www.r-project.org).

Results: a total of 260 patients were enrolled, of whom 185 had at least 12 months of follow-up. The cohort of these 185 patients was composed by 156 (84.3\%) females, and the majority (92.4\%) were Caucasians. Mean diagnostic delay (from onset until diagnosis) was about 20 months; mean value of ECLAM and mean number of organ/system involvement were both associated with a shorter diagnostic delay. Following study entry, there was a marked improvement of the clinical and serologic parameters of the disease, and of disease activity. However, patient's quality of life showed a trend towards deterioration, and some cardiovascular risk factors significantly increased over the 12 months period. About a third of patients with active disease at study entry went into remission (ECLAM=0) during 12 months of follow-up. Negative predictors for remission were: oral ulcers $(\mathrm{OR}=0.19,95 \% \mathrm{Cl} 0.04-0.86, \mathrm{p}=0.03)$, arthritis (OR $=0.44,95 \% \mathrm{Cl} 0.22-0.89, \mathrm{p}=$ 0.02 ), low C4 (OR $=0.31,95 \% \mathrm{Cl} 0.12-0.79, \mathrm{p}=0.01$ ), anti-SSB (Ro) antibodies (OR = $0.21,95 \% \mathrm{Cl} 0.05-0.97, \mathrm{p}=0.04)$, and therapy with mycophenolate $(\mathrm{OR}==0.13,95 \%$ $\mathrm{Cl} 0.02-1, \mathrm{p}=0.05)$. Both at baseline and during follow-up there was a widespread use of glucocorticoids and hydroxychloroquine comparing with other immunosuppressive drugs. We analysed the subcohort of patients persistently antidsDNA negative, and found that they were affected by a milder disease, with less severe clinical manifestations, less hospitalizations and lower disease activity, when compared with anti-dsDNA positives. 
Conclusions: our data confirm that the diagnosis of SLE can be very difficult at disease onset, and that still we are facing an important diagnostic delay. Clinical symptoms and serological parameters improve during the first period after diagnosis, probably due to drug therapy. However, there is a widespread use of glucocorticoids, and this is probably the reason for the early significant increase of some cardiovascular risk factors. Efforts should be made in order to improve our ability to make the diagnosis in the early stage of the disease and to treat patients with less dangerous drugs. 
Systemic lupus erythematosus (SLE) is a disease characterized by a broad spectrum of clinical manifestations and a multitude of laboratory abnormalities. The complexity of the disease could also explain why it can be difficult to identify SLE patients in an early stage of the disease. In fact, there are no pathognomonic clinical or serological features which can help clinicians in making SLE diagnosis. Criteria for the classification of SLE were elaborated by the American College of Rheumatology (ACR) firstly in 1971, then revised in 1982, and then again in 1997 (1). The new SLICC classification criteria seem to perform better than the revised ACR criteria in terms of sensitivity (but not specificity) (2). The SLICC criteria are meant to be clinically more relevant, allowing the inclusion of more patients with clinically defined lupus than are included using the current ACR criteria. However, even the SLICC classification criteria are not diagnostic criteria, and cannot be applied to every individual case.

So, the diagnosis of SLE remains very difficult, especially in the early stage of the disease, when it is more important to start the correct treatment, since it is well known that the delay in treatment is associated with a worse prognosis (3-5), and the progressive decrease in the time elapsed between disease onset and diagnosis is one of the major contributors to the improvement of survival (6) and quality of life (7) in SLE patients over time.

In 2012 we started a multicentre, prospective study on patients with recent (less than 12 months) onset SLE coming from nine Italian centres, with the purpose of collecting information on the clinical and laboratory characteristics of SLE patients at the beginning of the disease and during the first years of follow-up. Data on the clinical and serologic characteristics of this cohort at the time of patients' enrollment have already been published (8). In this paper we describe the clinical and serological features obtained after a oneyear follow-up period of this inception cohort and make comparison with the information obtained at the enrollment. In addition, we provide information on factors affecting the diagnostic delay of patients enrolled, on remission at the 12 month follow-up visit and factors correlated, on drug therapy both at baseline and follow-up, and on the differences between patients persistently anti-dsDNA negative with patients anti-dsDNA positive.

\section{Patients and methods}

This is a multicentre prospective study. Nine Italian centres with longstanding experience in Lupus management are involved. All patients with a diagnosis of SLE according to the 1997 American College of Rheumatology Classification Criteria (1) and a disease duration (from diagnosis until study entry) lower than 12 months were consecutively enrolled in the study. The study started on $1^{\text {st }}$ January, 2012. In this paper we report on patients enrolled until the end of June, 2017. 
Information on demographic characteristics, medical history, clinical symptoms, physical examinations, laboratory results, disease activity, disease damage, patient's quality of life, at the entry into the study and then every 6 months were recorded. Study data were collected and managed using REDCap electronic data capture tools hosted at the Italian Society for Rheumatology (9).

Global SLE disease activity was measured by the ECLAM, a validated measure of disease activity in SLE $(10,11)$. Cumulative damage was scored according to the SLICC Damage Index, a validated measure to assess damage in SLE (12). Patient's quality of life was estimated by means of a Visual Analogue Scale.

\section{Autoantibody assessment}

Autoantibodies were measured locally in each participating centre. The following autoantibodies were considered in this study: ANA, anti-dsDNA, anti-SSA (Ro), antiSSB (La), anti-Sm, anti-RNP, anticardiolipin (aCL), anti-beta2 glycoprotein I (antibeta2GPI), Lupus Anticoagulant (LA). ANA were measured by immunofluorescence using Hep2 cells as substrate. Anti-dsDNA were measured either by immunofluorescence using Crithidia luciliae or Farr technique. Anti-SSA, anti-SSB, anti-Sm, anti-RNP were measured either by immunoblot technique or ELISA. Anticardiolipin antibodies and anti-beta2GPI were measured by ELISA. Lupus anticoagulant was measured by coagulometric assay. Concerning $\mathrm{aCL}$ and antibeta2GPI patients were considered to be positive when either IgG or IgM (or both) were present at medium-high titer.

\section{Statistical analysis}

Results of the analysis of continuous variables are indicated as mean +/- standard deviation or median and range, as appropriate. Conventional chi-square and Fisher exact test were used for analyzing qualitative differences between independent samples. McNemar test was used for analyzing qualitative differences between patients at enrolment and after a one-year follow-up period. Student's t-test and Wilcoxon test were used for analyzing mean differences. A $p$ value $<0.05$ was taken to indicate statistical significance. The relationship between clinical and therapeutic variables with 12-months remission according to ECLAM was analysed by logistic model, and results presented as odds ratios (OR) and 95\% confidence intervals (Cl). All the analyses were performed using $R$ statistical environment (The $R$ project for statistical computing, https://www.r-project.org).

\section{Results}

At the end of June, 2017, 260 patients were enrolled, of whom 185 had at least 12 months of follow-up. The cohort of these 185 patients was composed by 156 $(84.3 \%)$ females, and the majority (92.4\%) were Caucasians. Mean age (SD) of 
patients at study entry was 37.92 (15.01) years, mean age at disease onset (first symptom of SLE) was 36.01 (15) years, mean age at diagnosis was 37.73 (14.99) years. Mean disease duration (from diagnosis until study entry) was 3.24 (4.16) months. Demographic features of this cohort are more extensively depicted in table 1.

\section{Diagnostic delay (from first symptom to diagnosis of SLE)}

Mean diagnostic delay (from onset until diagnosis) was about 20 months for the cohort of 185 patients. There were $126(68.48 \%)$ patients with a diagnostic delay less than a year, $41(22.28 \%)$ with a diagnostic delay between 1 and 5 years, and 17 $(9.24 \%)$ with a diagnostic delay greater than 5 years. Then, we analyzed the impact of the clinical and laboratory characteristics on the diagnostic delay. We found that mean value of ECLAM and mean number of organ/system involvement were both associated with a shorter diagnostic delay in the three groups $(p=0.02$ and $p=0.01$, respectively). On the contrary, diagnostic delay was not associated with age, gender, familiarity for SLE, pattern of clinical presentation.

No single ACR criteria as a first symptom of SLE was associated with the delay in diagnosis.

However, two patterns of combined organ/system involvement were found to be associated with longer diagnostic delay: patients with concomitant musculoskeletal and cardiorespiratory involvement $(p=0.05)$ and those with concomitant mucocutaneous and haematological involvement $(p=0.03)$.

In addition, we analysed the frequency of SLE autoantibodies in the various combined organ/system pattern of presentation of the disease. Anti-RNP antibodies were significantly more represented in patients presenting with concomitant mucocutaneous, musculoskeletal and haematological symptoms $(53.8 \%$ vs $19.6 \%$ in patients with other patterns of presentation, $p=0.009$ ). Anticardiolipin antibodies (medium-high titer) were significantly increased in those presenting with concomitant musculoskeletal and haematological symptoms (60\% vs $26.9 \%$ in patients with other patterns of presentation, $p=0.03$ ).

Cumulative frequency of the manifestations included in the ACR classification criteria until study entry and during 12 months of follow-up

The cumulative frequency (patients who ever had the symptom) until enrollment and during 12 months of follow-up of the manifestations included in the ACR classification criteria is reported in fig. 1. Comparing with study entry, there was an awaited increase of the frequency of all these manifestations, and this was statistically significant for malar rash $(p=0.02)$. 
The combined frequency of the mucocutaneous classification criteria was $54 \%$ at study entry and $58.2 \%$ at the end of the 12 month follow-up period, confirming that they are among the most common clinical manifestation included in the ACR classification criteria both at SLE onset and during the first 12 months of disease.

ANA were present in all but 2 patients at enrollment, both having anti-Ro antibodies.

Frequency of clinical symptoms and immunological features at study entry and during 12 months of follow-up

The frequency of the clinical and serological features of this cohort of patients at the enrollment visit and during the first 12 months after study entry is reported in Fig. 2 and Fig. 3. Following study entry, there was a marked improvement of the disease: patients underwent a significant decrease in all the clinical manifestations, C3 and C4 level significantly improved and anti-dsDNA antibody positive patients significantly decreased.

In agreement, median (interquartile range) ECLAM score decreased from 2.5 (1$4.5)$ at study entry to $1(0-2)$ at the 12 month visit $(p<0.001)$. However, median (interquartile range) patient's quality of life (VAS) decreased, albeit not significantly, from $54(40-70)$ to $50(30-71.5, p=0.43)$.

Mean number (SD) of hospitalizations decreased from 1.3 (1.2) until study entry to 0.5 (1.7) during the first 12 months after study entry $(p<0.001)$.

Forty-nine (32.9\%) patients with active disease at study entry went into remission $(E C L A M=0)$ during 12 months of follow-up. Overall, at 12 months $59(35.3 \%)$ of patients were in remission, according to ECLAM value $=0$. Negative predictors for remission were: oral ulcers ( $\mathrm{OR}=0.19,95 \% \mathrm{Cl} 0.04-0.86, \mathrm{p}=0.03)$, arthritis (OR = $0.44,95 \% \mathrm{Cl} 0.22-0.89, \mathrm{p}=0.02)$, low C4 (OR $=0.31,95 \% \mathrm{Cl} 0.12-0.79, \mathrm{p}=0.01$ ), anti-SSB (Ro) antibodies (OR $=0.21,95 \% \mathrm{Cl} 0.05-0.97, \mathrm{p}=0.04)$, and therapy with mycophenolate $(\mathrm{OR}==0.13,95 \% \mathrm{Cl} 0.02-1, \mathrm{p}=0.05)$.

We had 82 patients without clinical symptoms at the 12 month follow-up visit. Among them, according to the definition of remission proposed by Doria et al (13), no patient was in complete remission (no clinical symptom, normal serology, no prednisone, no immunosuppressants). Eight patients were in clinical remission without prednisone (no clinical symptom, abnormal serology, immunosuppressants allowed). Forty patients were in clinical remission with no more than $5 \mathrm{mg}$ prednisone daily (no clinical symptom, abnormal serology, immunosuppressants allowed), whereas 21 patients in clinical remission were taking more than $5 \mathrm{mg}$ prednisone daily. Therteen/82 patients were not valuable for missing informations. 
Cardiovascular risk factors were increased at 12 months: mean (SD) weight raised from $62.66(14.3)$ to $63.63(14.46) \mathrm{Kg}, \mathrm{p}<0.001$, mean (SD) BMI raised from 23 (4.26) to 23.36 (4.25), $p<0.001$, and mean (SD) waist circumference raised from 76.74 (12.08) to $79.32(12.33) \mathrm{cm}, \mathrm{p}=0.47$. Furthermore, obese patients raised from $5.9 \%$ to $17.7 \%(p=0.001)$ and those with dyslipidemia (blood cholesterol and/or triglycerides above upper normal limits) raised from $13.5 \%$ to $22.1 \%(p=0.004)$.

Drug therapy at study entry and during 12 months of follow-up

Drug therapy at study entry and during 12 months of follow-up is reported in Fig. 4. It is evident the widespread use of glucocorticoids in lupus patients both at study entry (85.4\%) and at follow-up (89.2\%) comparing with other immunosuppressive drugs. Hydroxychloroquine was given to a high proportion of patients too $(64.9 \%$ at study entry and $76.5 \%$ at follow-up, $p=0.004)$. Comparing with baseline, there was a significant increase of patients taking Azathioprine and Mycophenolate (10.8\% vs $18 \%, p=0.02$ and $10.3 \%$ vs $22.2 \%, p=0.003$, respectively).

Patients in remission at the 12 month follow-up visit were taking glucocorticoids (93.2\%), Hydroxychloroquine (81.4\%), and less frequently Azathioprine (11.9\%), Mycophenolate (18.6\%), Cyclophosphamide (8.5\%), Methotrexate (8.5\%), Cyclosporin A (5.1\%).

\section{$\underline{\text { SLE patients persistently anti-dsDNA negative }}$}

There were 21 patients who were persistently anti-dsDNA antibody negative. Even if they represent a small subcohort, we looked at possible differences with SLE patients who were anti-dsDNA positive at least once between baseline and the 12 month follow-up visit. The two subgroups had similar socio-demographic characteristics and similar frequency of the various comorbidities. However, patients persistently anti-dsDNA negative had more often discoid rash and photosensitivity both at baseline and during follow-up $(p<0.001)$, whereas they had less renal involvement at follow-up $(p=0.03)$, less reduction of $\mathrm{C} 3$ both at baseline and during follow-up $(p=0.01)$, and were less likely to be treated with prednisone both at baseline $(p=0.03)$ and during follow-up $(p=0.01)$ when comparing with anti-dsDNA positives (table 2).

Baseline disease activity was higher, albeit not significantly, in anti-dsDNA positive patients [ECLAM 3.3 (2.5) vs 2.4 (1.6)]. Accordingly, anti-dsDNA positive patients were more likely to be hospitalized during the follow-up period [mean number of hospitalizations $0.6(1.9)$ in anti-dsDNA positives vs $0.05(0.2)$ in anti-dsDNA 
negatives, $p=0.05]$. During follow-up, mean number of pregnancies was higher in anti-dsDNA negative patients [1.3 (1.1)] when comparing with anti-dsDNA positive patients [0.8 (1.2), $p=0.03$ ].

\section{Discussion}

The diagnosis of SLE remains very difficult, especially in the early stage of the disease, when it is more important to start the correct treatment, since it is well known that the delay in treatment is associated with a worse prognosis (3-5), and the progressive decrease in the time elapsed between disease onset and diagnosis is one of the major contributors to the improvement of survival (6) and quality of life (7) in SLE patients over time.

With the purpose of collecting information on the clinical and laboratory characteristics of SLE patients at the beginning of the disease and during the first years of follow-up, on behalf of the Italian Society of Rheumatology, a multicentre, prospective study on patients with recent (less than 12 months) onset SLE coming from nine Italian centres was started in January, 2012. Before starting the study, we discussed whether to consider the date of diagnosis as the time when patients reached the minimum for being classified as affected by SLE (according to ACR criteria), or the time when the "clinical" diagnosis had been made. In order to collect a more homogeneous cohort we choosed to consider as the date of diagnosis the time of classification (four or more ACR classification criteria).

Preliminary data on the clinical and serologic characteristics of this cohort at the time of patients' enrollment have already been published (8).

In this paper a further analysis on the 185 patients who had at least 12 months of follow-up is reported. Our data show that the mean diagnostic delay (from the appearance of the first symptom until the diagnosis) was about 20 months in this cohort. The majority (68.5\%) of patients were diagnosed no later than 12 months, $22.3 \%$ had the diagnosis in a period ranging from 1 to 5 years and $9.2 \%$ after 5 years after the appearance of the first symptoms. Number of organ/system involved and disease activity (ECLAM) at onset were significantly associated with an early recognition of disease ( $p=0.01$ and 0.02 , respectively). On the contrary, patients with contemporary mucocutaneous and haematological involvement and those with the combination of musculoskeletal and cardiorespiratory involvement experienced a significant delay in the diagnosis ( $p=0.02$ and 0.05 , respectively). These findings may suggest that the disease is more easily recognizable when more active and involving more organ/systems. On the other hand, the correct diagnosis can be missed when there is little awareness of the disease and when the patient is not seen by a multidisciplinary team devoted to SLE management. 
The most frequent symptoms at presentations were arthritis, mucocutaneous and haematological involvement. Malar rash and discoid lesions, that are more specific for SLE, were observed in less than a third of cases. Thus, the majority of patients present with poorly specific symptoms, making more difficult a prompt correct diagnosis.

When comparing baseline with 12 month follow-up data, it appears that clinical manifestations and disease activity laboratory indexes all decreased significantly. Accordingly, there was a significant decrease in disease activity and in the number of hospitalizations. However, therapy was not effective in improving the quality of life, that showed a trend towards deterioration, suggesting that much efforts should be done in improving our understanding of the needs of our patients.

In addition, cardiovascular risk factors such as obesity and dyslipidemia showed a significant increase during the 12 month follow-up period, and glucocorticoids were still taken by the large majority of patients at the 12 month follow-up visit, in contrast with the marked amelioration of disease symptoms and laboratory indexes.

Cardiovascular risk factors and glucocorticoids are both associated with a worse prognosis in lupus patients, being negative factors for long term morbidity and mortality (14-16). By contrast, hydroxychloroquine seems to have a beneficial effect on damage accrual and long-term prognosis in $\operatorname{SLE}(17,18)$, and the monoclonal antibody Belimumab is associated, among other beneficial effects, with steroid sparing $(19,20)$. In table 6 the therapy of SLE patients from other observational lupus cohorts is reported, confirming that glucocorticoids are too much frequently given to lupus patients across several countries. These data suggest that clinicians should assess frequently and carefully their therapeutic strategy in lupus patients, trying to avoid or minimize steroid usage and taking advantage from safer drugs.

The definition of remission is a difficult task in SLE, and studies on this argument are currently ongoing (26-29). When talking about remission in SLE, one should take into account clinical parameters, serologic parameters, therapy and duration. However, for the purpose of this study we defined a patient in remission when ECLAM was " 0 ". About a third of our patients were in remission (ECLAM activity index $=0$ ) at the 12 month follow-up visit. Baseline negative factors for remission were the presence of oral ulcers, arthritis, low C4 levels, anti-SSB (La) antibodies and being on mycophenolate.

A group of patients was persistently anti-dsDNA negative. When comparing with anti-dsDNA positive patients, they had a less severe disease, both at baseline and during follow-up, thus confirming that anti-dsDNA antibody is a useful marker for discriminating patients with a more aggressive disease (30). 
This study has the following limitations: 1) patients have been enrolled only in Rheumatological centres, thus conferring a possible selection bias; 2 ) laboratory examinations were not centralized; 3) some differences with other early Lupus cohorts, for instance in the time period for the definition of "early" disease and in the indexes utilized for measuring disease activity, make less reliable the comparison of our data with those from others.

In conclusion, even if relevant progress in SLE diagnosis has been made in the past decades, mainly because of the widespread introduction of the ANA test by indirect immunofluorescence during the early eighties, a significant number of patients is experiencing a delay in diagnosis greater than 12 months, and no significant reduction of the time elapsing from onset and diagnosis has been obtained in the recent years.

The complex etiopathogenesis and heterogeneous clinical manifestations make SLE commonly misdiagnosed. This diversity is a reason why a treatment may have beneficial effects in only a subset of patients. Identifying new SLE biomarkers might be a useful tool to sub-classify patients, quantify the risk of organ involvement and predict which clinical manifestation they might develop, as well as assign them the most appropriate treatment. It may also facilitate early and accurate SLE diagnosis and improve the evaluation of medications in clinical trials. Therefore, finding new specific biomarkers is very important and there is constant effort to obtain new and more valid tests for a better management of the disease.

As a consequence, many researchers are focusing on the discovery of new reliable biomarkers for the individuation of early and very early disease.

Studies of SLE immunopathogenesis have contributed to impressive advances in defining important mechanisms of disease. The role of the innate immune response, particularly the type I interferon pathway; the contribution of important $T$ cell subsets to altered immune regulation; and identification of genetic polymorphisms associated with disease were particularly noted, but significant areas of opportunity remain. Epigenetic analyses could provide a tool to understand the contribution of environmental factors to disease development and flares, and incorporating studies of metabolism was identified as an as yet untapped research opportunity. Study of well-phenotyped patients to relate the underlying biology of lupus to defined clinical subsets is an obvious approach that will require dedicated infrastructure and collaboration among clinicians and investigators.

Our data show that the cumulative frequency of the characteristics included in the current classification criteria is changing over time, and this should be taken into account when elaborating classification criteria. Efforts are being made in order to develop new classification criteria focused on the early stage of SLE, with the 
purpose of shortening the time from SLE onset and disease classification. In fact, new weighted classification criteria have been set up by an international collaborative study endorsed by ACR and EULAR, with the inclusion of data from the present cohort, among others (31). These new criteria have been presented at the 2017 ACR Congress. 


\section{References}

1) Hochberg MC. Updating the American College of Rheumatology revised criteria for the classification of systemic lupus erythematosus. Arthritis Rheum. 1997;40:1725.

2) Petri M, Orbai A-M, Alarcon GS, Gordon C, Merrill JT, Fortin PR, et al. Derivation and Validation of the Systemic Lupus International Collaborating Clinics Classification Criteria for Systemic Lupus Erythematosus. Arthritis Rheum 2012;64:2677-2686.

3) Esdaile JM, Joseph L, MacKenzie T, Kashgarian M, Hayslett JP. The benefit of early treatment with immunosuppressive agents in lupus nephritis. J Rheumatol 1994;21(11):2046-51.

4) Faurschou $M$, Starklint $H$, Halberg $P$, Jacobsen S. Prognostic factors in lupus nephritis: diagnostic and therapeutic delay increases the risk of terminal renal failure. J Rheumatol 2006;33(8):1563-9.

5) Ciruelo E, De la Cruz J, López I, Gómez-Reino JJ. Cumulative rate of relapse of lupus nephritis after successful treatment with cyclophosphamide. Arthritis Rheum 1996;39(12):2028-34.

6) Doria A, laccarino L, Ghirardello A, Zampieri S, Arienti S, Sarzi-Puttini P, et al. Longterm prognosis and causes of death in systemic lupus erythematosus. Am J Med 2006;119:1497-9.

7) Doria A, Rinaldi S, Ermani M, Salaffi F, laccarino L, Ghirardello A, et al. Healthrelated quality of life in Italian patients with systemic lupus erythematosus. II. Role of clinical, immunological, and psychological determinants. Rheumatology 2004;43:1580-6.

8) Sebastiani GD, Prevete I, Piga M, Iuliano A, Bettio S, Bortoluzzi A, et al. Early Lupus Project - A multicentre Italian study on systemic lupus erythematosus of recent onset. Lupus 2015;24:1276-82.

9) Harris PA, Taylor R, Thielke R, Payne J, Gonzalez N, Conde JG. Research electronic data capture (REDCap) - A metadata-driven methodology and workflow process for providing translational research informatics support. J Biomed Inform. 2009;42:37781. 
10) Vitali C, Bencivelli W, Isenberg DA, Smolen JS, Snaith ML, Sciuto M, Neri R, Bombardieri S. Disease activity in systemic lupus erythematosus: report of the Consensus Study Group of the European Workshop for Rheumatology Research. II. Identification of the variables indicative of disease activity and their use in the development of an activity score. The European Consensus Study Group for Disease Activity in SLE. Clin Exp Rheumatol. 1992;10:541-7.

11) Mosca M, Bencivelli W, Vitali C, Carrai P, Neri R, Bombardieri S. The validity of the ECLAM index for the retrospective evaluation of disease activity in systemic lupus rythematosus. Lupus. 2000;9:445-50.

12) Gladman D, Ginzler E, Goldsmith C, Fortin P, Liang $M$, Urowitz $M$, Bacon $P$, Bombardieri S, Hanly J, Hay E, Isenberg D, Jones J, Kalunian K, Maddison P, Nived O, Petri M, Richter M, Sanchez-Guerrero J, Snaith M, Sturfelt G, Symmons D, Zoma A. The development and initial validation of the Systemic Lupus International collaborating clinics/American college of rheumatology damage index for systemic lupus erythematosus. Arthritis Rheum. 1996;39:363-9.

13) Zen $M$, laccarino L, Gatto $M$, Bettio $S$, Nalotto L, Ghirardello A, Punzi L, Doria A. Prolonged remission in caucasian patients with SLE: prevalence and outcomes. Ann Rheum Dis 2015;74:2117-22.

14) Rizk A, Gheita TA, Nassef $S$, Abdallah A. The impact of obesity in systemic lupus erythematosus on disease parameters, quality of life, functional capacity and the risk of atherosclerosis. Int J Rheum Dis. 2012;15:261-7.

15) Sherer $Y$, Zinger $H$, Shoenfeld $Y$. Atherosclerosis in systemic lupus erythematosus. Autoimmunity. 2010;43:98-102.

16) Kalunian KC, Kim M, Xie X, Baskaran A, Daly RP, Merrill JT. Impact of standard of care treatments and disease variables on outcomes in systemic lupus erythematosus trials: analysis from the Lupus Foundation of America Collective Data Analysis Initiative. Eur J Rheumatol. 2016;3:13-19.

17) Akhavan PS, Su J, Lou W, Gladman DD, Urowitz MB, Fortin PR. The early protective effect of hydroxychloroquine on the risk of cumulative damage in patients with systemic lupus erythematosus. J Rheumatol. 2013;40:831-41.

18) Costedoat-Chalumeau N, Galicier L, Aumaître O, Francès C, Le Guern V, Lioté F, et al. Hydroxychloroquine in systemic lupus erythematosus: results of a French multicentre controlled trial (PLUS Study). Ann Rheum Dis. 2013;72:1786-92.

19) Schwarting A, Dooley MA, Roth DA, Edwards L, Thompson A, Wilson B. Impact of concomitant medication use on belimumab efficacy and safety in patients with systemic lupus erythematosus. Lupus. 2016 Dec;25:1587-1596. 
20) laccarino $L$, Bettio $S$, Reggia $R$, Zen $M$, Frassi $M$, Andreoli $L$, et al. Effects of Belimumab on Flare Rate and Expected Damage Progression in Patients With Active Systemic Lupus Erythematosus. Arthritis Care Res 2017;69:115-123.

21) Alarcón GS, McGwin G Jr, Bastian HM, Roseman J, Lisse J, Fessler BJ, Friedman AW, Reveille JD. Systemic lupus erythematosus in three ethnic groups. VII [correction of VIII]. Predictors of early mortality in the LUMINA cohort. LUMINA Study Group. Arthritis Rheum. 2001;45:191-202.

22) Urowitz MB, Gladman DD, Ibañez D, Fortin PR, Bae SC, Gordon C, et al. Evolution of disease burden over five years in a multicenter inception systemic lupus erythematosus cohort. Arthritis Care Res (Hoboken) 2012;64:132-7.

23) Nossent J, Kiss E, Rozman B, Pokorny G, Vlachoyiannopoulos P, Olesinska M, et al. Disease activity and damage accrual during the early disease course in a multinational inception cohort of patients with systemic lupus erythematosus Lupus 2010;19:949-56.

24) Parker B, Urowitz MB, Gladman DD, Lunt M, Donn R, Bae SC, et al. Impact of early disease factors on metabolic syndrome in systemic lupus erythematosus: data from an international inception cohort. Ann Rheum Dis. 2015;74:1530-6.

25) Ruiz-Irastorza G, García M, Espinosa G, Cabezas-Rodríguez I, Mitjavila F, González-León R, et al. Patterns of drug therapy in newly diagnosed Spanish patients with systemic lupus erythematosus. Clin Exp Rheumatol. 2016;34:466-72.

26) van Vollenhoven RF, Mosca M, Bertsias G, Isenberg D, Kuhn A, Lerstrøm K, et al. Treat-to-target in systemic lupus erythematosus: recommendations from an international task force. Ann Rheum Dis. 2014;73:958-67.

27) van Vollenhoven RF, Voskuyl A, Morand E, Aranow C. Remission in SLE: closing in on the target. Ann Rheum Dis. 2015;74:2103-6.

28) Zen $M$, laccarino L, Gatto $M$, Bettio $S$, Nalotto $L$, Ghirardello A, et al. Prolonged remission in Caucasian patients with SLE: prevalence and outcomes. Ann Rheum Dis. 2015;74:2117-22.

29) Doria A, Gatto M, laccarino L, Punzi L. Value and goals of treat-to-target in systemic lupus erythematosus: knowledge and foresight. Lupus. 2015;24:507-15. 
30) Pisetsky DS. Anti-DNA antibodies--quintessential biomarkers of SLE. Nat Rev Rheumatol. 2016;12:102-10.

31) Touma Z, Cervera R, Brinks R, Tani C, Hoyer BF, Costenbader KH, Lorenzoni V, Sebastiani G, Navarra SV, Bonfa E, Ramsey-Goldman R, Tedeschi SK, Doerner T, Johnson S, Aringer M, Mosca M. Associations Among Classification Criteria Items within Systemic Lupus Erythematosus [abstract]. Arthritis Rheumatol. 2017; 69 (suppl 10).

Acknowledgwments -

This work was done with the support of "Centro Studi SIR", the Epidemiologic Unit of Società Italiana di Reumatologia.

This work was supported by a specific grant from the national lupus patient association Gruppo LES Italiano - ONLUS. 
Table 1 - Baseline characteristics of the 185 patients with recent onset SLE

\begin{tabular}{|l|l|}
\hline Variables & values \\
\hline SOCIODEMOGRAPHIC & \\
\hline Female gender - n (\%) & $156(84.32 \%)$ \\
\hline Ethnicity - n (\%) & \\
\hline Caucasian & $171(92.43 \%)$ \\
\hline African & $5(2.7 \%)$ \\
\hline Indian & $1(0.54 \%)$ \\
\hline Arabic & $1(0.54 \%)$ \\
\hline Chinese & $1(0.54 \%)$ \\
\hline Other Asian & $2(1.08 \%)$ \\
\hline Other & $4(2.16 \%)$ \\
\hline Age at onset in years (first symptom/s of SLE) - mean (SD) & $36.01( \pm 15)$ \\
\hline Age at diagnosis in years (fulfillment of ACR criteria) - mean (SD) & $37.73( \pm 14.99)$ \\
\hline Age at enrollment in years - mean (SD) & $37.92( \pm 15.01)$ \\
\hline Disease duration in months (from diagnosis until enrollment) - mean (SD) $)$ & $3.24( \pm 4.16)$ \\
\hline Marital status - n (\%) & \\
\hline Married & $95(51.63 \%)$ \\
\hline Single & $58(31.52 \%)$ \\
\hline Widowed & $3(1.63 \%)$ \\
\hline Divorced & $0(0 \%)$ \\
\hline Separated & $1(0.54 \%)$ \\
\hline Partner & $27(14.67 \%)$ \\
\hline Years of school education - n (\%) & \\
\hline <= 8 years & $51(28.81 \%)$ \\
\hline $9-13$ years & $78(44.07 \%)$ \\
\hline$>13$ years & $48(27.12 \%)$ \\
\hline Socio economic status - n (\%) & $23(13.22 \%)$ \\
\hline Poor & $140(80.46 \%)$ \\
\hline Medium & $11(6.32 \%)$ \\
\hline High & $81(43.78 \%)$ \\
\hline Smoking - n (\%) & $51(62.96 \%)$ \\
\hline Ever smoked & $15(8.11 \%)$ \\
\hline of which Actually smoking $\%)$ \\
\hline Family history of SLE - n (\%) & \\
\hline
\end{tabular}


Table 2 - Cumulative frequency of selected clinical and serologic manifestations in patients persistently anti-dsDNA negative compared with anti-dsDNA positives

\begin{tabular}{|c|c|c|c|c|c|c|}
\hline & \multicolumn{3}{|c|}{ Baseline } & \multicolumn{3}{c|}{ 12 month follow-up } \\
\hline manifestation $-\mathbf{n}(\%)$ & Anti-DNA NEG & $\begin{array}{c}\text { anti-DNA } \\
\text { POS }\end{array}$ & p & Anti-DNA NEG & $\begin{array}{c}\text { anti-DNA } \\
\text { POS }\end{array}$ & p \\
\hline Malar rash & $8(38.1 \%)$ & $47(31.33 \%)$ & 0.62 & $9(42.86 \%)$ & $52(39.39 \%)$ & 0.81 \\
\hline Discoid rash & $5(23.81 \%)$ & $5(3.36 \%)$ & $<0.001$ & $6(30 \%)$ & $6(4.76 \%)$ & $<0.001$ \\
\hline Photosensitivity & $14(66.67 \%)$ & $35(23.49 \%)$ & $<0.001$ & $14(66.67 \%)$ & $39(29.55 \%)$ & $<0.001$ \\
\hline Oral ulcers & $3(14.29 \%)$ & $20(13.51 \%)$ & 1 & $3(14.29 \%)$ & $20(15.62 \%)$ & 1 \\
\hline Arthritis & $11(52.38 \%)$ & $99(66 \%)$ & 0.23 & $11(52.38 \%)$ & $104(73.24 \%)$ & 0.07 \\
\hline Serositis & $5(23.81 \%)$ & $37(24.67 \%)$ & 1 & $5(23.81 \%)$ & $39(29.32 \%)$ & 0.8 \\
\hline Renal disorder & $3(14.29 \%)$ & $50(33.33 \%)$ & 0.08 & $3(14.29 \%)$ & $53(39.85 \%)$ & 0.03 \\
\hline Neurological & $0(0 \%)$ & $13(8.67 \%)$ & 0.37 & $0(0 \%)$ & $16(12.6 \%)$ & 0.13 \\
\hline Hematological & $10(47.62 \%)$ & $81(54 \%)$ & 0.64 & $10(47.62 \%)$ & $86(62.77 \%)$ & 0.23 \\
\hline Low C3 & $9(42.86 \%)$ & $106(72.6 \%)$ & 0.01 & $5(25 \%)$ & $71(56.35 \%)$ & 0.01 \\
\hline Low C4 & $15(71.43 \%)$ & $129(88.36 \%)$ & 0.05 & $15(75 \%)$ & $95(75.4 \%)$ & 1 \\
\hline anti-SSA $($ Ro) & $10(52.63 \%)$ & $51(36.69 \%)$ & 0.21 & $5(62.5 \%)$ & $29(40.28 \%)$ & 0.27 \\
\hline anti-SSB $($ La) & $5(26.32 \%)$ & $19(13.77 \%)$ & 0.17 & $2(25 \%)$ & $9(12.5 \%)$ & 0.3 \\
\hline anti-nRNP & $8(42.11 \%)$ & $26(18.98 \%)$ & 0.03 & $1(12.5 \%)$ & $18(25.35 \%)$ & 0.67 \\
\hline anti-Sm & $3(15.79 \%)$ & $28(20.29 \%)$ & 0.77 & $1(12.5 \%)$ & $12(16.67 \%)$ & 1 \\
\hline anticardiolipin & $4(22.22 \%)$ & $36(27.91 \%)$ & 0.78 & $3(42.86 \%)$ & $13(20.31 \%)$ & 0.18 \\
\hline anti-beta2GPI & $2(11.11 \%)$ & $27(21.95 \%)$ & 0.37 & $2(28.57 \%)$ & $9(14.52 \%)$ & 0.31 \\
\hline Lupus anticoagulant & $3(16.67 \%)$ & $25(19.69 \%)$ & 1 & $1(14.29 \%)$ & $9(14.06 \%)$ & 1 \\
\hline
\end{tabular}


Table 3 - Therapy in SLE observational Lupus cohorts

\begin{tabular}{|c|c|c|c|c|c|c|}
\hline Author/year/ref & n. patients & $\begin{array}{c}\text { time of } \\
\text { treatment }\end{array}$ & $\begin{array}{l}\text { disease } \\
\text { activity }\end{array}$ & oral GC* & antimalarials & IS§ \\
\hline Alarcon/2001/20 & 229 & enrollment & 10.8 (SLAM) & $89 \%$ & $56 \%$ & $24 \%$ \\
\hline $\begin{array}{c}\text { Nossent } / 2010 / 2 \\
1\end{array}$ & 200 & $\begin{array}{l}1 \text { yr after } \\
\text { diagnosis }\end{array}$ & 12.2 (SLEDAI) & $83 \%$ & $46 \%$ & $60 \%$ \\
\hline $\begin{array}{c}\text { Urowitz/2012/2 } \\
2\end{array}$ & 389 & enrollment & 9.6 (SLEDAI) & $63 \%$ & $46 \%$ & $31 \%$ \\
\hline Parker/2015/23 & 1150 & enrollment & 5.4 (SLEDAI) & $69 \%$ & $66 \%$ & $40 \%$ \\
\hline $\begin{array}{c}\text { Ruiz- } \\
\text { Irastorza/2016/2 } \\
4 \\
\end{array}$ & 223 & $\begin{array}{l}1 \text { yr after } \\
\text { diagnosis }\end{array}$ & 9.8 (SLEDAI) & $65 \%$ & $81 \%$ & $39 \%$ \\
\hline $\begin{array}{c}\text { Early SLE Italian } \\
\text { cohort/2017 }\end{array}$ & 185 & $\begin{array}{l}1 \text { yr after } \\
\text { diagnosis }\end{array}$ & 1.1 (ECLAM) & $89 \%$ & $76 \%$ & $60 \%$ \\
\hline
\end{tabular}

*oral GC: oral glucocorticoids. §IS: immunosuppressants 
Fig. 1 - Cumulative frequency of the manifestations included in the ACR classification criteria until study entry and during 12 months of follow-up in the cohort of 185 patients with recent onset SLE
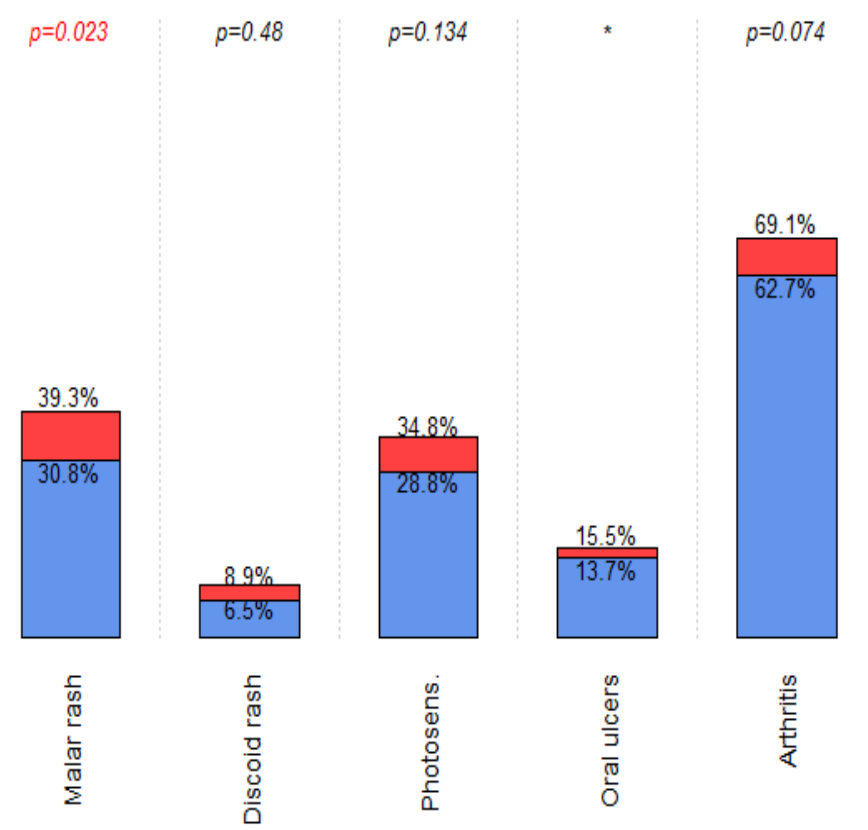

$p=0.48$

$p=0.248$

$p=0.248$
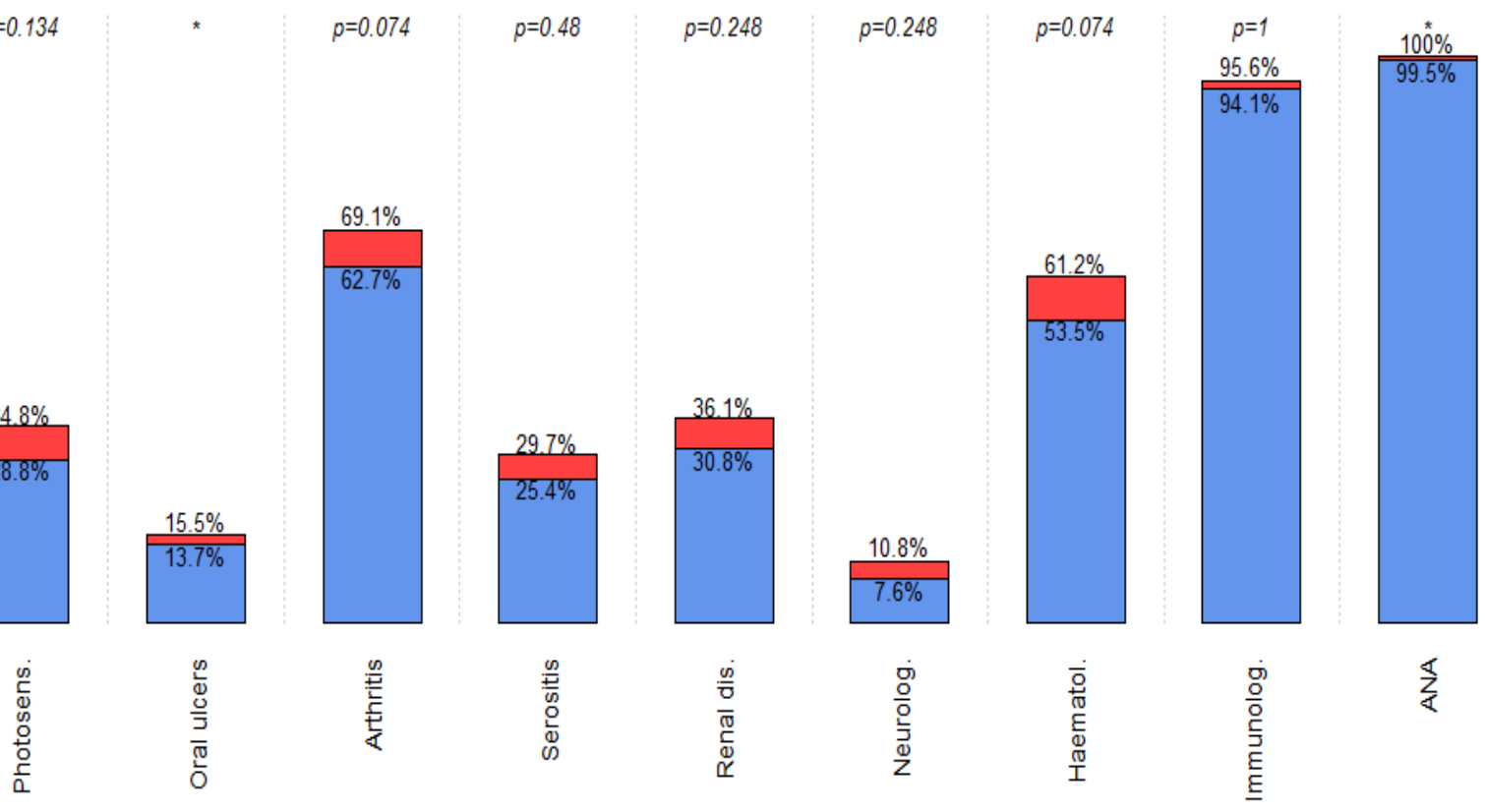

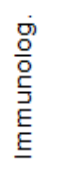

*It's impossible to calculate McNemar test's p-value, since there is at least one cell of the frequency table (useful for calculating pvalue) equal to zero. 
Fig. 3 - Frequency (\%) of immunological features at study entry and during 12 months of follow-up in the cohort of 185 patients with recent onset SLE

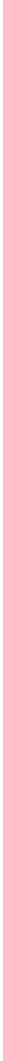


